\title{
Discrete-Time Exponentially Stabilizing Fuzzy Sliding Mode Control via Lyapunov's Method
}

\author{
Radiša Ž. Jovanović and Zoran M. Bučevac \\ Faculty of Mechanical Engineering, University of Belgrade, 16 Kraljice Marije, 11120 Belgrade 35, Serbia \\ Correspondence should be addressed to Radiša Ž. Jovanović; rjovanovic@mas.bg.ac.rs
}

Received 30 July 2014; Revised 7 February 2015; Accepted 8 February 2015

Academic Editor: Rustom M. Mamlook

Copyright (c) 2015 R. Ž. Jovanović and Z. M. Bučevac. This is an open access article distributed under the Creative Commons Attribution License, which permits unrestricted use, distribution, and reproduction in any medium, provided the original work is properly cited.

\begin{abstract}
The exponentially stabilizing state feedback control algorithm is developed by Lyapunov's second method leading to the variable structure system with chattering free sliding modes. Linear time-invariant discrete-time second order plant is considered and the control law is obtained by using a simple fuzzy controller. The analytical structure of the proposed controller is derived and used to prove exponential stability of sliding subspace. Essentially, the control algorithm drives the system from an arbitrary initial state to a prescribed so-called sliding subspace $S$, in finite time and with prescribed velocity estimate. Inside the sliding subspace $S$, the system is switched to the sliding mode regime and stays in it forever. The proposed algorithm is tested on the real system in practice, DC servo motor, and simulation and experimental results are given.
\end{abstract}

\section{Introduction}

It is well-known that the variable structure control systems (VSCS) theory has been existing for several decades. Almost everything published in the area is related to the VSCS with sliding modes, with known features and advantages. Until the mid-1980s, the results concerned exclusively the continuous time type of these systems. With the development of computer and digital technology, the discrete-time version of the problem has become more important in the academic research and industries. Discrete-time sliding mode control is an attempt to eliminate the problems caused by the discretization of continuous-time controllers. Great attention was directed to the existence of sliding mode regime inside the so-called sliding subspace and finite system state reaching time to the sliding subspace in this type of systems, because it is quite different from its continuous counterpart. Although discrete-time VSCS with sliding mode is characterized by a phenomenon that the actual so-called sliding control is applied inside sliding subspace (see, e.g., [1-3]), the chattering problem was raised. Many different ways were proposed to reduce it or fully eliminate it [4]. On the other side, system convergence to the sliding subspace conditions was dealt with, where analogy to the continuous systems conditions was massively exploited.

Many of the authors treated special classes of the systems, most frequently, linear systems, systems in companion canonical form, and nonlinear systems, with Lyapunov's second method as a powerful design and analysis technique $[5,6]$. There are no papers with exponential stability of sliding subspace, except that some authors speak about exponential changes of, in general case, vector variable, usually designated, for example, by $S$ and used for sliding subspace definition as $S=0$; see [7], among others. In [8] authors spoke about exponential state vector changes on the way towards zero origin of a ball.

Recently, the integration of fuzzy techniques and conventional control approaches has been an active research focus. Fuzzy controllers are inherently nonlinear controllers, and the major advantage of fuzzy control technology over the traditional control technology is its capability of capturing and utilizing qualitative human experience and knowledge in a quantitative manner through the use of fuzzy sets, fuzzy rules, and fuzzy logic [9]. Based on the differences of fuzzy control rules, approaches to fuzzy logic control can be roughly classified into the conventional fuzzy control 
and Takagi-Sugeno (T-S) model based fuzzy control. The conventional fuzzy control systems are essentially heuristic and model free and various approaches have been developed for stability analysis. The key idea of these approaches is to regard a fuzzy controller as a nonlinear controller and embed the stability and/or control design problem of fuzzy control systems into conventional nonlinear system stability theory $[10,11]$. T-S models are based on using a set of fuzzy rules to describe a global nonlinear system in terms of a set of local linear models which are smoothly connected by fuzzy membership functions, and they provide a basis for development of systematic approaches to stability analysis and controller design of fuzzy control systems in view of powerful conventional control theory and techniques [12-14].

Combining fuzzy logic (FL) and sliding mode control (SMC) theory, so-called fuzzy sliding mode control (FSMC) has the advantages of both SMC and FLC. The analogy between a simple and a sliding-mode controller with a boundary layer is shown in [15]. In [16, 17], for example, fuzzy controllers are designed to satisfy the sliding conditions, and in these approaches, the focus is on the design of sliding surface via the fuzzy logic theory. Some fuzzy control rules to construct reaching control under the assumption that equivalent control already exists is used in [18]. A fuzzymodel-based controller which guarantees the stability of the closed loop controlled system is suggested in [19], where the closed-loop system consists of the TS fuzzy model and the switching type fuzzy-model-based controller. Fuzzy logic has been also utilized to adapt the parameters of SMC to achieve a better performance and especially attenuate the chattering of control input.

The above works are related to continuous systems, and discrete systems were much less considered in the context of FSMC. The design of the fuzzy sliding mode control to meet the requirement of necessary and sufficient reaching conditions of sliding mode control of discrete nonlinear system is considered in [20]. A robust controller based on the sliding mode and the dynamic T-S fuzzy state model for discrete systems is developed in [21].

One motivation for this work stems from the fact that discrete-time type of the problem clearly makes place of microprocessor compensator application in the systems. The main motivation for the paper to be related to the exponential stability of the FSMC, as a higher quality stability property, goes from the fact that, in most of papers, only stability of sliding subspace $S$ was considered.

Following Hui and Żak's [3] conditions for chattering free sliding mode, in this paper, the exponential stabilizing fuzzy control algorithm is developed by Lyapunov's second method leading to the variable structure system with chattering free sliding modes, for linear time-invariant second order discrete-time system. That is, the exponential stability to be in exact sense related to the sliding subspace as a set, defined over working point distance from the set, during its approaching to the set. In the work, essentially, the objective is to push the system state from an arbitrary initial position to the sliding subspace $S$, in finite time, and with estimate of approaching velocity defined by an exponential law. The velocity could not be smaller than the exponential law defines. Once the system reaches $S$ it stays there forever working in sliding mode regime, which is chattering free, as well, and with the system state asymptotic approaching to the zero equilibrium state. The control law is obtained by using a simple fuzzy controller and the analytical structure of the proposed controller is derived and used in the proof of the theorem on exponential stability of sliding subspace.

\section{Problem Statement, Notation, and Some Definitions}

Linear time-invariant second order discrete-time system is considered, which is described by its state equation:

$$
x(k+1)=A x(k)+b u(k)
$$

where $k \in N_{0}, N_{0}=\{0,1,2, \ldots\}, x(k)$ is state vector at time $k$, $u(k) \in R$ is control vector at time $k, A$ and $b$ are real constant matrix and vector of appropriate dimensions, respectively, and $(A, b)$ is assumed to be a controllable pair.

For the system (1), define a hyperplane

$$
c x=0,
$$

where $c=\left(\begin{array}{ll}c_{1} & c_{2}\end{array}\right)$ is a constant nonzero row vector. The hyperplane is the so-called sliding subspace $S$ (in further text only $S$ ). Clearly, for second order system $S$ is sliding line, described by

$$
S=\{x: c x=0\}
$$

Also, $c b \neq 0$ is assumed.

The objective of this paper is to develop variable structure type of state feedback control law:

$$
u=u(x)
$$

based on fuzzy logic, which guarantees that the state $\chi(k ; x(0) ; u(\cdot))$ of the system (1) reaches $S$ in finite time and with velocity whose estimate is defined by an exponential law. Once $S$ has been reached, the controller is required to keep the state within it thereafter, which means positive invariance of $S$ relative to the system motion and what is denoted as sliding mode regime. During this regime, inside $S$, convergence to zero equilibrium with prescribed mode $\lambda$ can be guaranteed if the $c$ has been appropriately chosen. For $c$ choice, in general case, see [22].

More rigorously, $S$ is positive invariant relative to the system (1) motion if and only if $x(0) \in S$ implies $\chi(k ; x(0) ; u(\cdot)) \epsilon$ $S, \forall k \in N_{0}$.

Furthermore, some other notations and definitions are given for the reason of their usage in theorems, which are the main results. Real $n$-dimensional state space $R^{n}$ is with Euclidean norm denoted by $\|\cdot\|$. For $S$,

$$
d(x, S)=\inf (\|x-y\|: y \in S)
$$

is the distance between $S$ and the point $x \in R^{n}$.

Definition 1. The state $x=0$ of the system (1), (2) is stable in $S$ (with respect to $S$ ) iff $\forall \varepsilon \in R_{+},\left(R_{+}=\right] 0,+\infty[$ ), $\exists \delta=\delta(\varepsilon) \in R_{+}$such that $x(0) \in S$ and $\|x(0)\|<\delta(\varepsilon)$ 
implies that $\chi(k ; x(0) ; u(\cdot))$ exists, $\|\chi(k ; x(0) ; u(\cdot))\|<\varepsilon$, and $\chi(k ; x(0) ; u(\cdot)) \in S, \forall k \geq 0$.

Definition 2. The state $x=0$ of the system (1), (2) is attractive (globally) in $S$ (with respect to $S$ ) iff $\exists \Delta>0(\Delta=\infty)$ such that $x(0) \in S$ and $\|x(0)\|<\Delta$ implies that $\chi(k ; x(0) ; u(\cdot))$ exists, $\lim \{\|\chi(k ; x(0) ; u(\cdot))\|: k \rightarrow+\infty\}=0$, and $\chi(k ; x(0) ; u(\cdot)) \in$ $S, \forall k \in N_{0}$.

Definition 3. The state $x=0$ of the system (1), (2) is (globally) asymptotically stable in $S$ (with respect to $S$ ) iff it is both stable and (globally) attractive in $S$.

Definition 4. The system (1), (2) is stable in $S$ (with respect to $S$ ) iff its state $x=0$ is globally asymptotically stable in $S$.

Definition 5. $S$ is exponentially (globally) stable, relative to the system (1), (2), iff $\exists \Delta \in] 0,+\infty[(\Delta=+\infty)$ and $\exists \alpha \in$ $[1,+\infty[$ and $\beta \in] 0,+\infty[$ such that distance $d[x(0), S]<\Delta$ implies that $\chi(k ; x(0) ; u(\cdot))$ exists and $d[\chi(k ; x(0) ; u(\cdot)), S] \leq$ $\alpha d[x(0), S] e^{-\beta k}, \forall k>0$.

Remark 6. Previous definitions are valid and in the general case, for $x \in R^{n}$.

The problem stated in the objective of the paper can be reformulated as follows: the objective of the paper is to develop the control law (4) such that $S$ is globally exponentially stable relative to the system (1), (2) and the system (1), (4) is stable in the sliding mode regime with appropriately prescribed modes. Control law (4) will be developed using fuzzy logic controller.

\section{Structure of FLC}

The key idea of fuzzy sliding mode control is to integrate fuzzy control and sliding mode control in such a way that the advantages of both techniques can be used. One approach is to design conventional fuzzy control systems, and sliding mode controller is used to determine best values for parameters in fuzzy control rules. Thereby, stability is guaranteed and robust performance of the closed-loop control systems is improved. In another approach, the control design is based on sliding mode techniques while the fuzzy controller is used as a complementary controller. Also, sliding mode control law can be directly substituted by a fuzzy controller.

Sliding mode controllers, generally, involve a discontinuous control action which often results in chattering phenomena due to imperfections in switching devices and delays. Commonly used methods for chattering elimination are to replace the relay control by a saturation function and boundary layer technique. In some applications of fuzzy sliding mode control, the continuous switching function of the boundary layer is replaced with equivalent fuzzy switching function.

In this paper, the fuzzy controller is used in the reaching phase. The controller should realize nonlinear control law which will guarantee exponential stability of sliding subspace $S$. This section introduces the principal structure of the proposed controller.

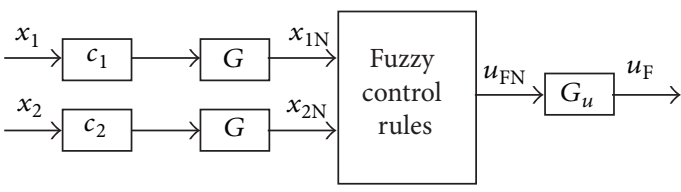

FIgURE 1: The proposed fuzzy logic controller.

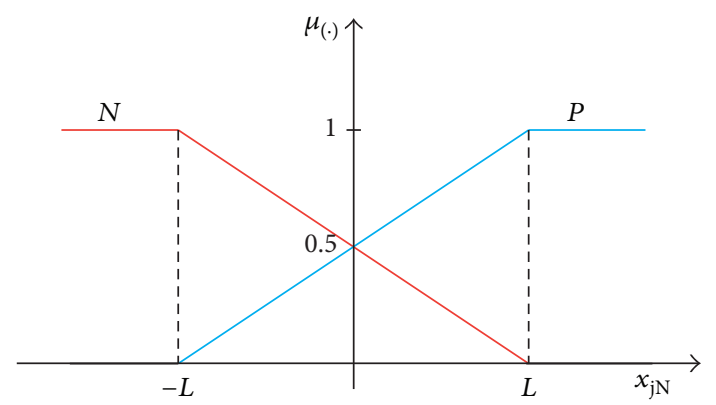

FIgURE 2: The input membership functions.

The fuzzy logic controller that will be evaluated is one of the simplest, Figure 1. It employs only two input variables, $x_{1}(k)$ and $x_{2}(k)$. Constant $c_{1}$ and $c_{2}$ are component of vector $c$ from (2).

Scaling Factors. The use of normalized domains requires a scale transformation, which maps the physical values of the input variables ( $x_{1}$ and $x_{2}$ in the present study) into a normalized domain. This is called input normalization. Furthermore, output denormalization maps the normalized value of the control output variable $\left(u_{\mathrm{FN}}\right)$ into its respective physical domain $\left(u_{\mathrm{F}}\right)$. The relationships between scaling factors $\left(G, G_{u}\right)$ and the input and output variables are as follows:

$$
\begin{aligned}
x_{1 \mathrm{~N}}(k) & =G \cdot c_{1} \cdot x_{1}(k), \\
x_{2 \mathrm{~N}}(k) & =G \cdot c_{2} \cdot x_{2}(k), \\
u_{\mathrm{F}}(k) & =G_{u} \cdot u_{\mathrm{FN}}(k) .
\end{aligned}
$$

Fuzzification Module. It converts instantaneous value of a process state variable into a linguistic value with the help of the represented fuzzy set. The parametric functional description of the triangular shaped membership function is the most economic one and hence it is considered here. The membership functions of input variables are shown in Figure 2.

Let $x_{j}^{*}$ be the one crisp input. Then, the fuzzified version of $x_{j}^{*}$ after normalization is its degree of membership in $\mu_{N}\left(x_{j \mathrm{~N}}^{*}\right)$ and $\mu_{P}\left(x_{j \mathrm{~N}}^{*}\right)$, where $N$ and $P$ are the linguistic values taken by $x_{j \mathrm{~N}}$. Here, symbols $N$ and $P$ have common meanings negative and positive, respectively.

Assumption 7. The value $x_{j \mathrm{~N}}(k)$ satisfies

$$
x_{j \mathrm{~N}}(k) \in[-L, L], \quad \forall j=1,2, \forall k \in N_{0} .
$$




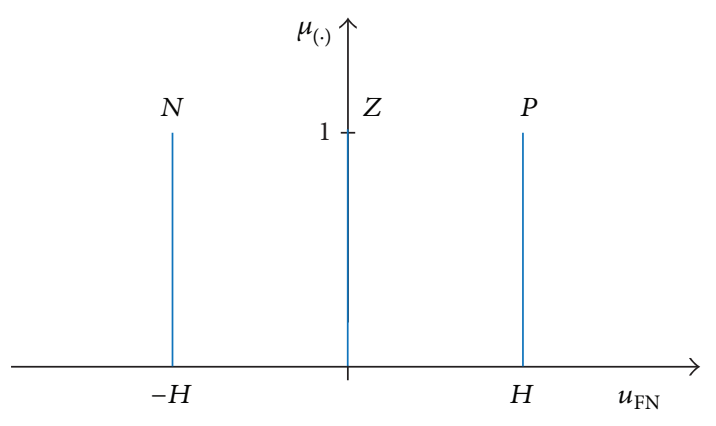

Figure 3: The output membership functions.

According to the previous assumption, mathematical description of the input membership functions is, respectively, given by

$$
\mu_{N}\left(x_{j \mathrm{~N}}\right)=\frac{-x_{j \mathrm{~N}}+L}{2 L}, \quad \mu_{P}\left(x_{j \mathrm{~N}}\right)=\frac{x_{j \mathrm{~N}}+L}{2 L}, \quad j=1,2 .
$$

Remark 8. It is noticed that

$$
\mu_{N}\left(x_{j \mathrm{~N}}\right)+\mu_{P}\left(x_{j \mathrm{~N}}\right)=1, \quad j=1,2 .
$$

The membership functions for the normalized output $\left(u_{\mathrm{FN}}\right)$ are singleton and are shown in Figure 3. In Figures 2 and 3, $L$ and $H$ are two positive constants chosen by the designer, which can be fixed after being determined.

Remark 9. To ensure that Assumption 7 is valid, one must be careful in choosing the scaling factor $G$. In practical implementation, the maximum and minimum values of variables $x_{1}$ and $x_{2}$ are known, and the factor $G$ is chosen so that it satisfies the following conditions:

$$
G \cdot c_{j} \cdot\left|x_{j}\right|_{\max } \leq L, \quad \forall j=1,2 .
$$

Remark 10. For a second order system, it is common to define the row vector $c$ as $c=\left(\begin{array}{ll}c_{1} & 1\end{array}\right)$.

Fuzzy Control Rules. Using the aforementioned membership functions, the following control rules are established for the fuzzy logic control part:

$$
\begin{aligned}
& R_{1}: \text { If } x_{1 \mathrm{~N}} \text { is } N \text { and } x_{2 \mathrm{~N}} \text { is } N \text { then } u_{\mathrm{FN}} \text { is } N \\
& R_{2}: \text { If } x_{1 \mathrm{~N}} \text { is } N \text { and } x_{2 \mathrm{~N}} \text { is } P \text { then } u_{\mathrm{FN}} \text { is } Z \\
& R_{3}: \text { If } x_{1 \mathrm{~N}} \text { is } P \text { and } x_{2 \mathrm{~N}} \text { is } N \text { then } u_{\mathrm{FN}} \text { is } Z \\
& R_{4}: \text { If } x_{1 \mathrm{~N}} \text { is } P \text { and } x_{2 \mathrm{~N}} \text { is } P \text { then } u_{\mathrm{FN}} \text { is } P .
\end{aligned}
$$

Inference Engine. The basic function of the inference engine is to compute the overall value of control output variable based on the individual contribution of each rule in the rule base. A degree of match for each rule is established by using the defined membership functions.
Here, the antecedent of each rule is evaluated by using the triangular norm $(t$-norm). The $t$-norm used in this paper is intersection (AND function), which is mathematically given as

$$
\mu_{m}\left(x_{1 \mathrm{~N}}, x_{2 \mathrm{~N}}\right)=T\left(\mu_{(\cdot)}\left(x_{1 \mathrm{~N}}\right), \mu_{(\cdot)}\left(x_{2 \mathrm{~N}}\right)\right), \quad(\cdot)=N, P .
$$

Then, based on this degree of match, the clipped fuzzy set representing the value of the control output variable is determined via Mamdani inference method. Thus, outcomes of fuzzy rules are

$$
\begin{gathered}
\mu_{\mathrm{CLm}}\left(u_{\mathrm{FN}}\right)=\min \left(\mu_{m}, \mu_{U m}\left(u_{\mathrm{FN}}\right)\right), \\
U_{m} \in\{N, Z, P), \quad m=1,2,3,4 .
\end{gathered}
$$

Finally, the clipped values for the control output of each rule, previously denoted by $\mu_{C L m}, m=1,2,3,4$, are aggregated, thus forming the value of the overall control output.

From the rule base it may be noted that the control rules $R_{2}$ and $R_{3}$ generate two memberships $\mu_{2}$ and $\mu_{3}$ which have the same output fuzzy set defined by $\mu_{Z}\left(u_{\mathrm{FN}}\right)$. For such situations a combined membership is obtained by using the triangular conorm ( $t$-conorm, or $s$-norm). The $t$-conorm considered for this study is Lukasiewicz OR $t$-conorm:

$$
\mu_{2,3}^{T C}=\min \left(1, \mu_{2}+\mu_{3}\right) .
$$

Defuzzification Module. Defuzzification module converts the set of modified control output values into a crisp value. Defuzzification is done using the well-known COS (center of sum) method. According to this method and taking into account the previously analyzed structure of the proposed fuzzy controller and the fact that the output fuzzy sets are singleton, the crisp value of control output is given by

$$
u_{\mathrm{FN}}=\frac{\mu_{1} \cdot(-H)+\mu_{2,3}^{T C} \cdot 0+\mu_{4} \cdot H}{\mu_{1}+\mu_{2,3}^{T C}+\mu_{4}} .
$$

Since for Lukasiewicz OR $t$-conorm it is valid that

$$
\mu_{2,3}^{T C}=\mu_{2}+\mu_{3} \leq 1
$$

(15) can be written as

$$
u_{\mathrm{FN}}=\frac{\mu_{1} \cdot(-H)+\mu_{2} \cdot 0+\mu_{3} \cdot 0+\mu_{4} \cdot H}{\mu_{1}+\mu_{2}+\mu_{3}+\mu_{4}} .
$$

To obtain analytical expression of the proposed controller, all combinations of input variables must be considered. If Assumption 7 holds, there are eight input combinations (IC's), as shown in Figure 4 . The control rules $R_{1}-R_{4}$ in 


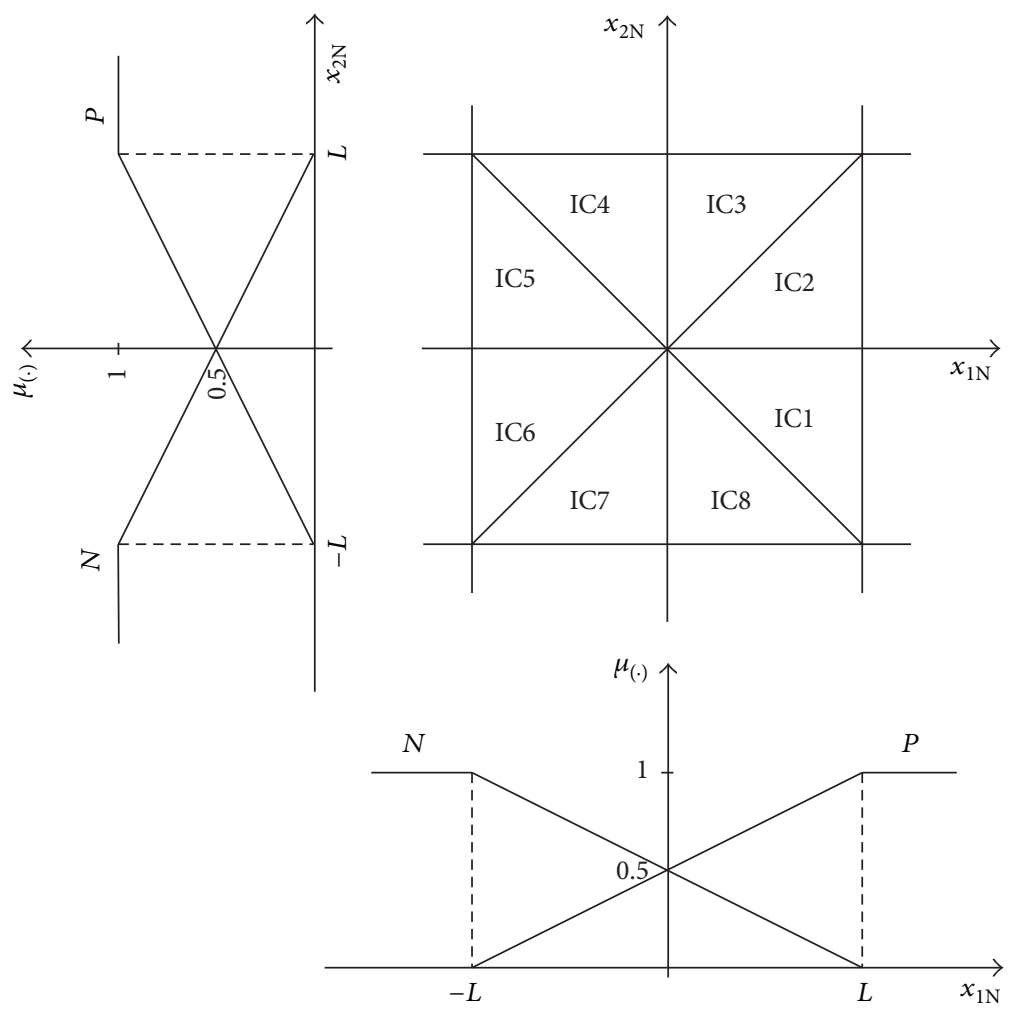

FIGURE 4: The regions of the fuzzy controller input values.

TABLE 1: Outcomes of fuzzy rules in IC regions.

\begin{tabular}{lcccc}
\hline IC & \multicolumn{4}{c}{ Fuzzy rules } \\
& $R_{1}$ & $R_{2}$ & $R_{3}$ & $R_{4}$ \\
\hline IC1 & $\mu_{N}\left(x_{1 \mathrm{~N}}\right)$ & $\mu_{N}\left(x_{1 \mathrm{~N}}\right)$ & $\mu_{N}\left(x_{2 \mathrm{~N}}\right)$ & $\mu_{P}\left(x_{2 \mathrm{~N}}\right)$ \\
IC2 & $\mu_{N}\left(x_{1 \mathrm{~N}}\right)$ & $\mu_{N}\left(x_{1 \mathrm{~N}}\right)$ & $\mu_{N}\left(x_{2 \mathrm{~N}}\right)$ & $\mu_{P}\left(x_{2 \mathrm{~N}}\right)$ \\
IC3 & $\mu_{N}\left(x_{2 \mathrm{~N}}\right)$ & $\mu_{N}\left(x_{1 \mathrm{~N}}\right)$ & $\mu_{N}\left(x_{2 \mathrm{~N}}\right)$ & $\mu_{P}\left(x_{1 \mathrm{~N}}\right)$ \\
IC4 & $\mu_{N}\left(x_{2 \mathrm{~N}}\right)$ & $\mu_{N}\left(x_{1 \mathrm{~N}}\right)$ & $\mu_{N}\left(x_{2 \mathrm{~N}}\right)$ & $\mu_{P}\left(x_{1 \mathrm{~N}}\right)$ \\
IC5 & $\mu_{N}\left(x_{2 \mathrm{~N}}\right)$ & $\mu_{P}\left(x_{2 \mathrm{~N}}\right)$ & $\mu_{P}\left(x_{1 \mathrm{~N}}\right)$ & $\mu_{P}\left(x_{1 \mathrm{~N}}\right)$ \\
IC6 & $\mu_{N}\left(x_{2 \mathrm{~N}}\right)$ & $\mu_{P}\left(x_{2 \mathrm{~N}}\right)$ & $\mu_{P}\left(x_{1 \mathrm{~N}}\right)$ & $\mu_{P}\left(x_{1 \mathrm{~N}}\right)$ \\
IC7 & $\mu_{N}\left(x_{1 \mathrm{~N}}\right)$ & $\mu_{P}\left(x_{2 \mathrm{~N}}\right)$ & $\mu_{P}\left(x_{1 \mathrm{~N}}\right)$ & $\mu_{P}\left(x_{2 \mathrm{~N}}\right)$ \\
IC8 & $\mu_{N}\left(x_{1 \mathrm{~N}}\right)$ & $\mu_{P}\left(x_{2 \mathrm{~N}}\right)$ & $\mu_{P}\left(x_{1 \mathrm{~N}}\right)$ & $\mu_{P}\left(x_{2 \mathrm{~N}}\right)$ \\
\hline
\end{tabular}

(11) are used to evaluate appropriate control law in each IC region, as in [23]. The results of evaluating the fuzzy control rules $R_{1}-R_{4}$ are given in Table 1 .

Applying (17) to the results from Table 1 and taking into account (6) and (8), analytical structure of the controller is easily obtained, as follows:

$u_{\mathrm{F}}(k)= \begin{cases}\frac{H G G_{u}\left(c_{1} x_{1}(k)+c_{2} x_{2}(k)\right)}{2\left(2 L-G c_{1}\left|x_{1}(k)\right|\right)}, & \text { IC1, IC2, IC5, IC6 } \\ \frac{H G G_{u}\left(c_{1} x_{1}(k)+c_{2} x_{2}(k)\right)}{2\left(2 L-G c_{2}\left|x_{2}(k)\right|\right)}, & \text { IC3, IC4, IC7, IC8. }\end{cases}$
Defining function $g\left(x_{1}, x_{2}\right)$,

$$
g\left(x_{1}, x_{2}\right)= \begin{cases}\frac{H G G_{u}}{2\left(2 L-G c_{1}\left|x_{1}(k)\right|\right)}, & \text { IC1, IC2, IC5, IC6 } \\ \frac{H G G_{u}}{2\left(2 L-G c_{2}\left|x_{2}(k)\right|\right)}, & \text { IC3, IC4, IC7, IC8. }\end{cases}
$$

(18) can be written as

$$
u_{\mathrm{F}}(k)=g\left(x_{1}, x_{2}\right) c x(k)
$$

It is obvious that the function $g\left(x_{1}, x_{2}\right)$ is a nonlinear function, with the following minimal and maximal values:

$$
g_{\min }=\frac{H G G_{u}}{4 L}, \quad g_{\max }=\frac{H G G_{u}}{2 L},
$$

and $g\left(x_{1}, x_{2}\right)>0, \forall x_{1}, x_{2} \in R$. One should not forget that parameter $G$ must satisfy Assumption 7. The range of the value of function $g\left(x_{1}, x_{2}\right)$ is determined by the choice of parameters $H, G, L$, and $G_{u}$. Without loss of generality, it can be assumed that $L=H=1$, and they are commonly normalized domain boundaries. One example of function $g\left(x_{1}, x_{2}\right)$ is shown in Figure 5.

\section{Main Results}

In this section, the solution of the problem stated in Section 2 is provided by the following theorems. 


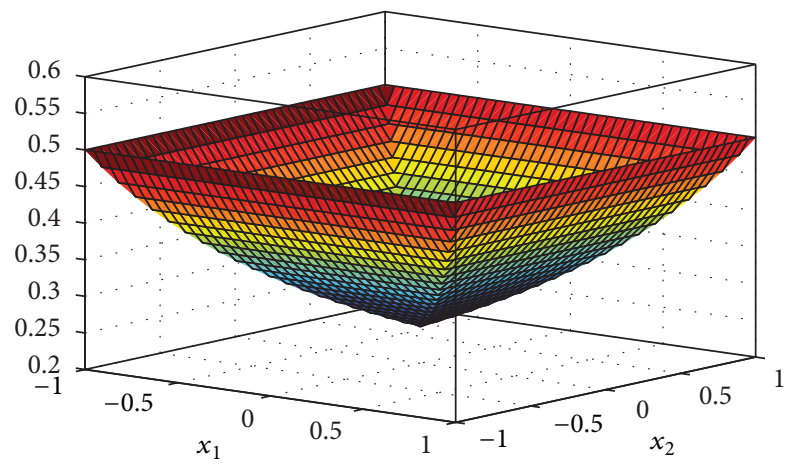

FIGURE 5: Example of function $g\left(x_{1}, x_{2}\right)$ for $L=H=1, G=G u=1$, and $c 1=c 2=1$.

\section{Theorem 11. Applying}

$$
u^{o s}(k)=-\frac{1}{c b} c A x(k)
$$

the so-called one step control, to the system (1), outside $S$, is necessary and sufficient for $x(k)$ to reach the $S$ in one sampling period (one step).

\section{Proof.}

Necessity. Let us assume that $x(k)$ of the system (1) is arbitrary, outside of sliding subspace, $x(k) \notin S$, and a control pushes it to the sliding subspace in one step (sampling period). It follows that $x(k+1) \in S$. That fact, description of $S$, and the system (1) state equation lead to

$$
c A x(k)+c b u(k)=0,
$$

and finally

$$
u(k)=u^{\mathrm{os}}(k)=-\frac{1}{c b} c A x(k) .
$$

Sufficiency. When the system state is outside of sliding subspace, $x(k) \notin S$, the action of one step control is assumed. State equation of the system (1) and expression of $S$ give

$$
\begin{aligned}
c x(k+1) & =c A x(k)+c b u^{o s}(k)=c A x(k)-c b \frac{1}{c b} c A x(k) \\
& =0,
\end{aligned}
$$

which means that $x(k+1) \in S$.

Remark 12. One step control can be encountered in VSCS papers, in different contexts; see, for example, $[3,4,13]$ among others.

\section{Theorem 13. Applying}

$$
u^{s l}(k)=-\frac{1}{c b} c A x(k),
$$

the actual so-called sliding control, to the system (1), inside $S$, is necessary and sufficient for $S$ to be positive invariant relative to the system (1) solution $\chi\left(k ; x(0) ; u^{s l}(k)\right)$.
Proof.

Necessity. Positive invariance of $S$ relative to the system (1) solution $\chi\left(k ; x(0) ; u^{\mathrm{sl}}(k)\right)$ is assumed. So, every time $x(k) \in S$, $x(k+1) \in S$ as well. From the previous sentence, description of $S$ and state equation of the system (1) follows that

$$
c A x(k)+c b u^{\mathrm{sl}}(k)=0,
$$

and further

$$
u^{\mathrm{sl}}(k)=-\frac{1}{c b} c A x(k)
$$

Sufficiency. Let us assume, relative to system $(1), x(k) \in S$ and control (26) is applied. Then, state equation of the system (1) and expression for $S$ give

$$
\begin{aligned}
c x(k+1) & =c A x(k)+c b u^{\mathrm{sl}}(k)=c A x(k)-c b \frac{1}{c b} c A x(k) \\
& =0,
\end{aligned}
$$

which implies that $x(k+1) \in S$.

Remark 14. One step control and sliding control formally are identical.

The application of actual sliding control $u^{\text {sl }}(k)$ (henceforth referred to as $u^{\text {sl }}(k)$ ) to the system (1) inside $S$ is the phenomenon of discrete-time VSCS with sliding modes. Using this fact and the intention for developing of the variable structure type of the controller for the system (1), the control (4) components could be more precisely specified as

$$
u[x(k)]= \begin{cases}u^{+}[x(k)], & c x(k)>0 \\ u^{\mathrm{sl}}[x(k)], & c x(k)=0 \\ u^{-}[x(k)], & c x(k)<0 .\end{cases}
$$

Contrary to the $u^{\mathrm{sl}}(k)$, which is applied to the system (1) inside $S$, let us refer to the control which is applied to the system (1) outside of $S$ as the outer control, $u^{\text {ot }}(k)$. The following theorems are basic for solving the stated problem by Lyapunov's second method.

Theorem 15. Let the system (1), (30) be considered. S is globally exponentially stable relative to the system (1), (30) if there exists scalar function $v$ and numbers $\left.\eta_{i} \in\right] 0,+\infty[, i=1,2,3$ such that
(a) $v(x) \in C\left(R^{2}\right)$,
(b) $\eta_{1} d(x, S) \leq v(x) \leq \eta_{2} d(x, S), \forall x \in\left(R^{2}\right)$,
(c) $\Delta v(x) \leq-\eta_{3} d(x, S), \forall x \in\left(R^{2}\right)$,

where $\Delta v(x)$ is the first forward finite difference of $v(x)$.

Proof. The proof starts with the assumption that sufficient conditions are fulfilled, while the exponential stability of $S$ should be shown. 
Condition (b) of the theorem implies

$$
-d[x(k), S] \leq \eta_{2}^{-1} v[x(k)],
$$

which with (c) give

$$
\Delta v[x(k)] \leq-\eta_{2}^{-1} \eta_{3} v[x(k)] .
$$

The last inequality, after solving, gives at first

$$
\begin{array}{r}
v[x(k)] \leq v[x(0)] e^{-\beta k}, \quad \beta=\ln \frac{\eta_{2}}{\eta_{2}-\eta_{3}}, \\
\eta_{2}>\eta_{3}, \quad \forall x(0) \in R^{2},
\end{array}
$$

and further by means of condition (a),

$$
d[x(k), S] \leq \alpha d[x(0), S] e^{-\beta k}, \quad \alpha=\eta_{1}^{-1} \eta_{2} .
$$

From (a), it is clear that $\eta_{1} \leq \eta_{2}$ which yields $\alpha \in[1,+\infty[$, and above relationship between $\eta_{2}$ and $\eta_{3}$ guarantee that $\beta \epsilon$ ] $0,+\infty$ [. In that way, it is shown that all stated by Definition 5 is fulfilled; that is, the exponential stability of $S$ is proved.

Remark 16. Previous theorem is valid and in the general case for $x \in R^{n}$.

Scalar function $v$ defined by

$$
v(x)=(\operatorname{sign} c x) c x
$$

as Lyapunov's function candidate and outer control law

$$
\begin{array}{r}
u^{\mathrm{ot}}(k)=-\frac{1}{c b}[c A x(k)-F(k) \operatorname{sign} c x(k)] \\
F(k) \in R, \quad F(k)>0, \quad \forall k \in N_{0}
\end{array}
$$

are chosen relative to the system (1). Evidently, such sort of $u^{\text {ot }}$ together with $u^{\text {sl }}$ which is unique and already stated represents the control of (30) type. The following theorems are basic for solving of the stated problem by Lyapunov's second method.

Theorem 17. $S$ is globally exponentially stable relative to the system (1) if the following control is applied:

$$
u(k)=\left\{\begin{array}{l}
u^{o t}(k)=-\frac{1}{c b}[c A x(k)-F(k) \operatorname{sign} c x(k)] \\
F(k)=|c x(k)|-\left|u_{F}(k)\right|, \\
u^{s l}(k)=-\frac{1}{c b}[c A x(k)]
\end{array}\right.
$$

with $u_{F}(k)$ defined by (19), (20) and with $g\left(x_{1}, x_{2}\right)<1$.

Proof. Previously introduced Lyapunov's function candidate fulfills evidently the conditions (a) of Theorem 15. Let us show that conditions (b) and (c) are fulfilled. Related to the condition (b) and taking into account that $|c x|=\zeta d$, where $\zeta$ is a constant and $d$ is distance between $x$ and hyperplane $c x=0$, it follows that

$$
v(x)=(\operatorname{sign} c x) c x=|c x|=\zeta d,
$$

and further

$$
\begin{gathered}
\eta_{1} d(x, S) \leq v(x) \leq \eta_{2} d(x, S), \\
\left.\eta_{1} \in\right] 0,+\infty\left[\leq \zeta, \quad \eta_{2} \in\right] 0,+\infty[\geq \zeta .
\end{gathered}
$$

State of system (1) at time $k$ is adopted to be out of $S$. Then

$$
\begin{aligned}
\Delta v[x(k)] & =v[x(k+1)]-v[x(k)] \\
& =F(k)|\operatorname{sign} c x(k)|-|c x(k)| \\
& =\left(|c x(k)|-\left|u_{\mathrm{F}}(k)\right|\right) \cdot|\operatorname{sign} c x(k)|-|c x(k)| \\
& =\left(1-g\left(x_{1}, x_{2}\right)\right)|c x(k)|-|c x(k)| \\
& =-g\left(x_{1}, x_{2}\right)|c x(k)|=-g\left(x_{1}, x_{2}\right) \zeta d[x(k), S] \\
& \leq-g_{\text {min }} \zeta d[x(k), S]=-\eta_{3} d[x(k), S]
\end{aligned}
$$

in which way the proof is finished.

According to Theorem 17,

$$
\Delta v[x(k)]=-g\left(x_{1}, x_{2}\right) v[x(k)]
$$

with $g\left(x_{1}, x_{2}\right)>0$ and $g\left(x_{1}, x_{2}\right)<1$, which leads to the following equation:

$$
\begin{array}{r}
v[x(k+1)]=\left(1-g\left(x_{1}, x_{2}\right)\right) v[x(k)]=\mu\left(x_{1}, x_{2}\right) v[x(k)], \\
\left.\mu\left(x_{1}, x_{2}\right) \in\right] 0,1[.
\end{array}
$$

From nonlinearity of the control low it is obvious that control gain is high when the system state is far from sliding surfaces and as small as possible in neighborhood of the sliding subspace. Also, it may be noted that $v[x(k)]$ increments, from step to step, that is, absolute value of $|c x|$, are getting smaller over time.

Function $v[x(k)]$ is a decreasing function along motion of the system, but it will never be zero. Obviously, the system (1) state never gets into $S$. Moreover, from (42), it is obvious that as the working point approaches the $S$, the value of the function $\mu\left(x_{1}, x_{2}\right)$ tends to its maximum value

$$
\mu_{\max }=\mu\left(x_{1}, x_{2}\right)_{\max }=1-g\left(x_{1}, x_{2}\right)_{\min } .
$$

Let $O_{\sigma}(S)$ be $\sigma$-neighborhood of $S$ and $v_{m \sigma}=\min \{v(x)$ : $x$ is such that $d(x, S)=\sigma\}$. To be guaranteed for $x(k)$ to enter $O_{\sigma}(S)$, settling time

$$
k_{s}=\left[0, \log _{\mu_{\max }} \frac{v_{m \sigma}}{v(0)}\right]+1
$$

is necessary to elapse, where $k_{s}=\left[0, \log _{\mu_{\max }}\left(v_{m \sigma} / v(0)\right)\right]$ is the biggest integer from the denoted segment. After the state reached $O_{\sigma}(S)$, one step control, $u^{\text {os }}$, should be applied to the system (1) in which way finite reaching time in $S$ for $x(k)$ is realized. The previous facts are summarized by the following theorem. 
Theorem 18. $S$ is globally exponentially stable relative to the system (1) with finite reaching time in $S$, if the following control is applied:

$$
u(k)=\left\{\begin{array}{l}
u^{o t}(k)=-\frac{1}{c b}[c A x(k)-F(k) \operatorname{sign} c x(k)] \\
F(k)=|c x(k)|-\left|u_{F}(k)\right|, \quad \forall k=0, \ldots, k_{s}-1 \\
u^{o t}\left(k_{s}\right)=u^{o s}\left(k_{s}\right)=-\frac{1}{c b}[c A x(k)] \\
u^{s l}(k)=-\frac{1}{c b}[c A x(k)], \quad \forall k=k_{s}+1, k_{s}+2, \ldots
\end{array}\right.
$$

Proof. Compared to the proof of Theorem 17, here the proof is the same.

\section{Simulation and Experimental Results}

The proposed algorithm was tested experimentally on a DC servo motor with the gear and load, whose linear mathematical model (without considering the major nonlinear effects: the speed dependent friction, dead zone, and backlash) is as follows:

$$
J \ddot{\theta}(t)+B \dot{\theta}(t)=\frac{\eta_{g} \eta_{m} k_{t} K_{g}}{R_{m}} U(t),
$$

where $\theta(t)$ is the angular position of the load shaft, $J$ is the total moment of inertia reflecting to the output shaft, $B$ is the viscous friction coefficient, and $U(t)$ is the motor input voltage. $R_{m}, k_{t}, \eta_{m}, \eta_{g}$, and $K_{g}$ are, respectively, the motor armature resistance, the motor torque constant, the motor efficiency, the gearbox efficiency, and the total gear ratio.

In the previous model, it is assumed that the motor inductance is much smaller than the resistance, so it is ignored, as the case with used servo motor is. The experiments were performed with the Quanser rotary servo motor, SRV02. This model is equipped with the optical encoder and tachometer, for motor position and speed measuring, respectively. Q8-USB data acquisition board for real-time data acquisition and control was used, with Matlab/Simulink software and QUARC@, real-time control. The parameters numerical values are as follows: $J=0.021\left(\mathrm{kgm}^{2}\right), B=$ $0.084(\mathrm{Nms} / \mathrm{rad}), R_{m}=2.6(\Omega), k_{t}=0.0077(\mathrm{Nm} / \mathrm{A}), \eta_{m}=$ $0.69, \eta_{g}=0.9$, and $K_{g}=70$.

Choosing $x_{1}=\theta$ and $x_{2}=\dot{\theta}$ as state variables and $u=$ $U(t)$ as control, at first, the continuous time state equation was obtained, and finally after applying an accurate discretization procedure with sampling period $T=0.01(\mathrm{~s})$, the discretetime state equation of the system was obtained as follows:

$$
x(k+1)=\left[\begin{array}{cc}
1 & 0.08266 \\
0 & 0.6746
\end{array}\right] x(k)+\left[\begin{array}{c}
0.002653 \\
0.4979
\end{array}\right] u(k) .
$$

The above mathematical model of real DC servo motor was derived as adequate discrete-time state equation. In the way the system matrix parameters $A$ and $b$ are available. Control algorithm from Theorem 18 is used to control the motor. For simulation and experiment, parameter $\sigma=0.005$

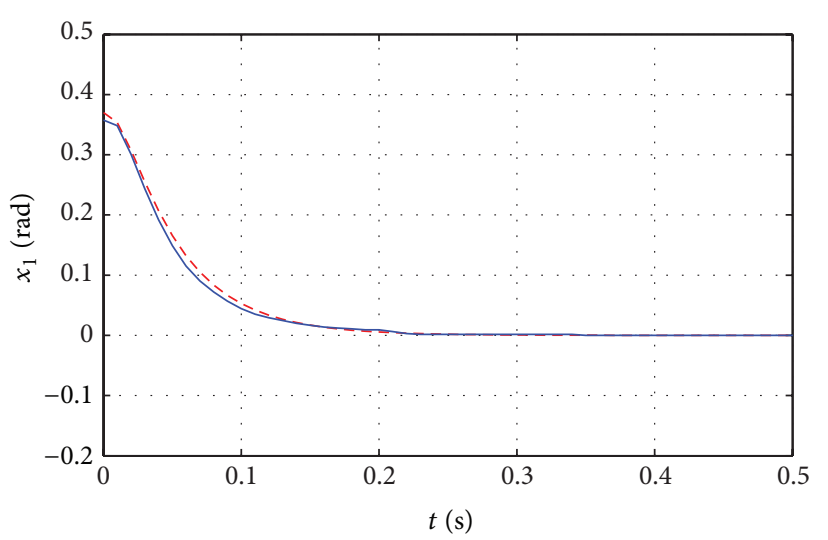

- - Simulation

- Experiment

FIGURE 6: The state variable $x_{1}$.

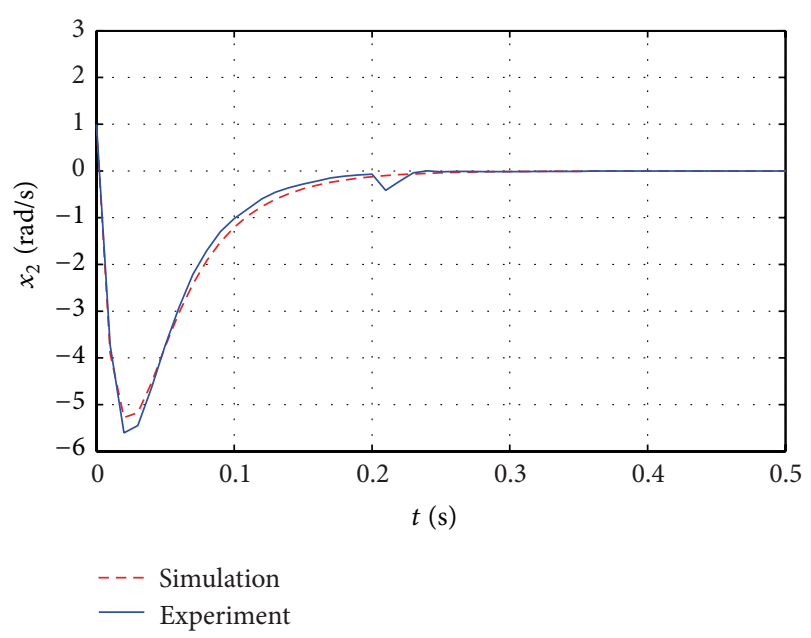

Figure 7: The state variable $x_{2}$.

is adopted which defines very narrow $\sigma$-neighborhood of sliding subspace $S$. Also, sampling time $T=0.01$ (s) and vector $c=\left(\begin{array}{ll}80 & 1\end{array}\right)$, determined so as the system is stable in sliding mode regime, are adopted which gives $\zeta=\|c\|=$ 80.006. Fuzzy logic controller is defined by $L=H=1$, $G=0.02$, and $G_{u}=40$, which implies $g_{\min }=0.2$ and $g_{\max }=0.4$. Taking into account estimate relationships, on one hand, between $\zeta$ and $\eta_{1}$ and $\eta_{2}$, and, on another hand, between $\zeta, g_{\min }$, and $\eta_{3}$, parameters $\eta_{1}=30, \eta_{2}=90$, and $\eta_{3}=15$ are chosen, giving parameters of exponential low $\alpha=3$ and $\beta=0.1823$. For initial state $x(0)=\left(\begin{array}{ll}0.36 & 1\end{array}\right)^{T}$, after calculation, $v_{m \sigma}=0.4, v(0)=29.8$, and settling time $k_{s}=20$ are obtained.

The parallel simulation and experimental results are shown, in Figures 6-11 related to the algorithm from Theorem 18. Figures 6 and 7 show the system state variables. The control signal that drives the state onto the sliding subspace is shown in Figure 9. The sliding function and distance are shown in Figures 8 and 10, respectively. From 


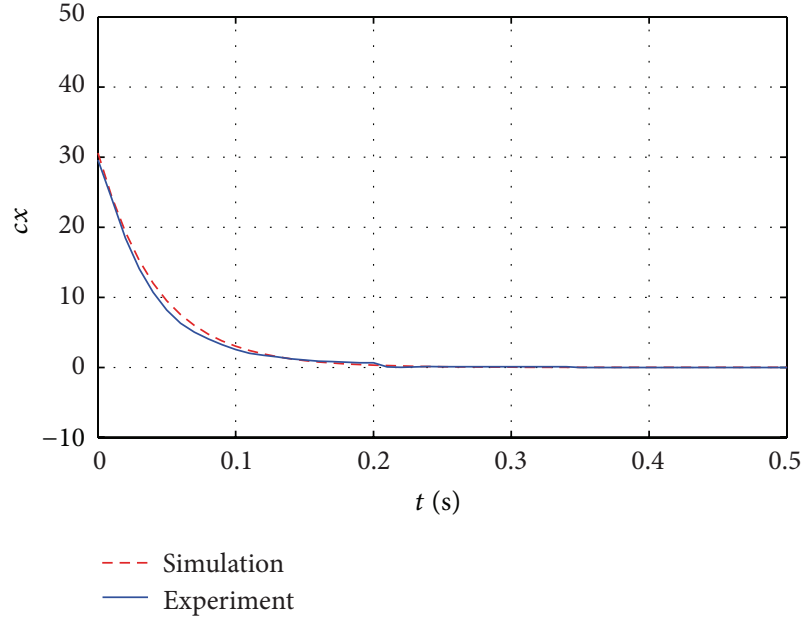

FIgURE 8: The sliding function $c x$.

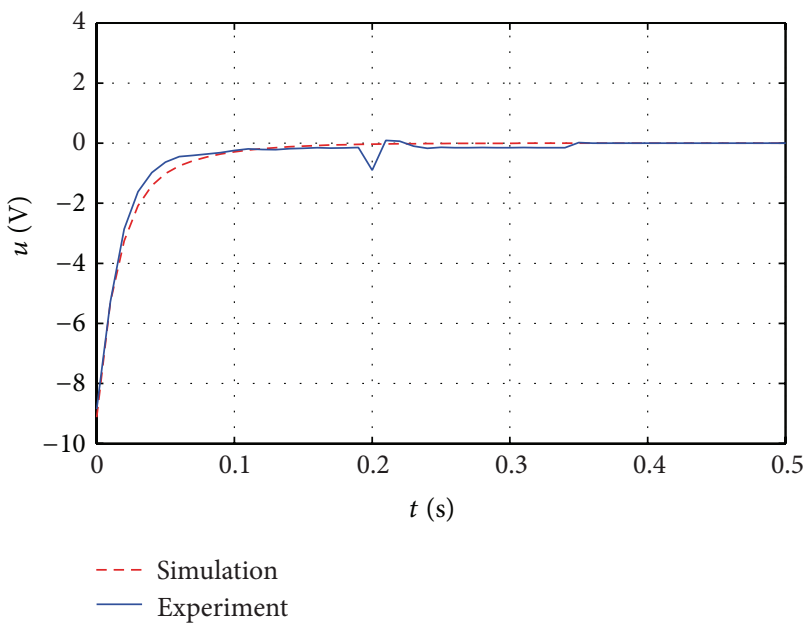

FIgURE 9: The control variable.

the figures, it could be seen that the system state from initial position approaches the sliding subspace $S$ but not slower than the exponential law defined by the parameters $\alpha$ and $\beta$. At instant $k_{s}=20(t=0.2 \mathrm{sec})$ the state for the first time enters the $\sigma$-neighborhood of $S$. If it continues in the same way, it will never reach $S$. To reach it, one step control is applied at $k_{s}$ driving state in one step to $S$, so the character of the system approaching $S$ changes at $k_{s}$. At $k_{s}+1=21$ the system state is in $S$ staying in it thereafter forever. Inside $S$ the system is stable too, so the system state asymptotically approaches the zero state. Simulation results fully confirm the theoretical results and meet the expectations of Theorems 15, 17 , and 18 .

The experimental results have demonstrated that the proposed control scheme is valid and effective for the real applications. It is shown that there is no chattering in sliding mode. However, in practice, errors could not be caused only by computation but also by measurement accuracy (resolution, sensitivity), noise existence, nonlinearity, and inadequate description of the real system by linear model as

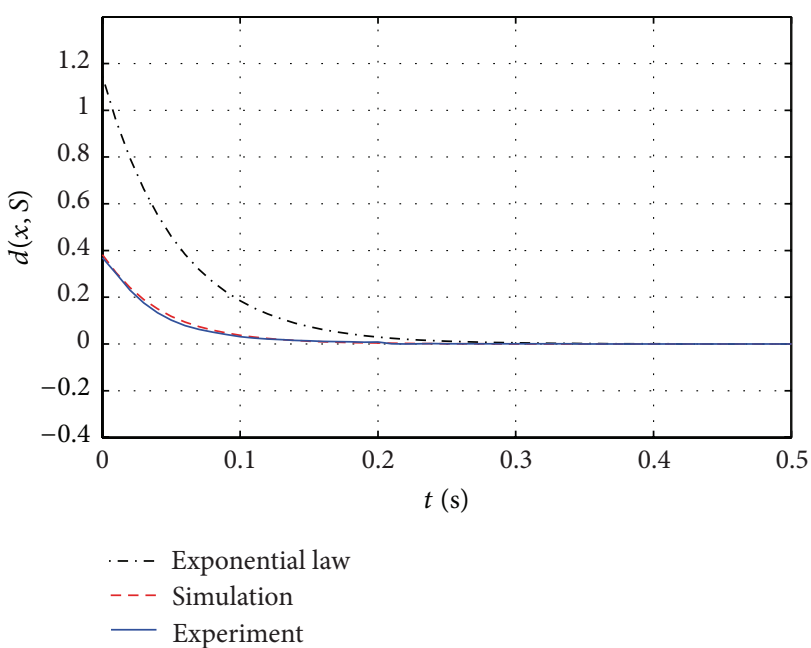

FIgURE 10: The distance.

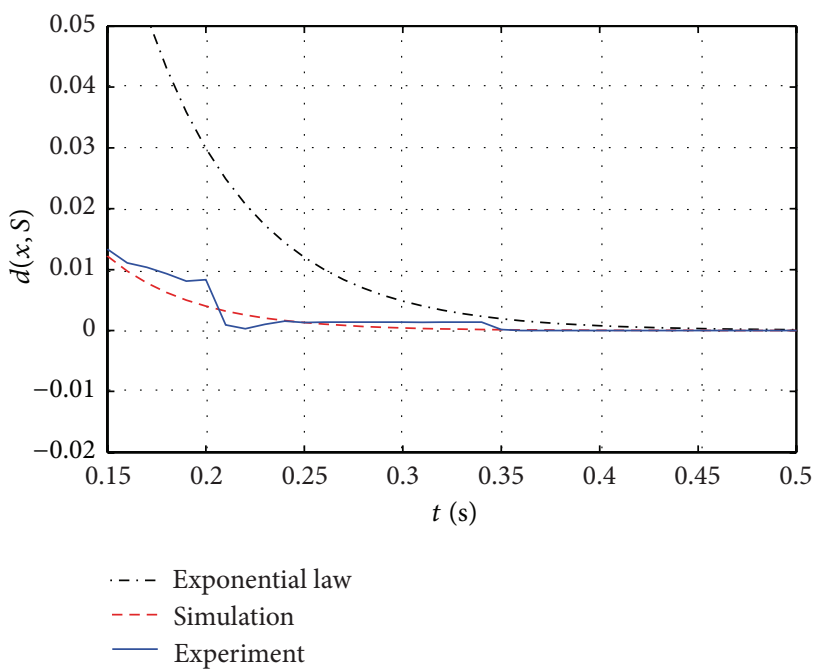

FIgURE 11: Augmented part of Figure 10.

well. All this could cause not entering the $\sigma$-neighborhood of sliding subspace exactly in step $k_{s}$ than at some later instant, which is the case here in the paper experimental application example. After entering the $\sigma$-neighborhood of $S$ at $k_{s}$ or not and applying one step control at $k_{s}$, a little bit of sliding subspace missing occurs. Here, that missing is a little bit more visible at the beginning of reaching the sliding subspace, but very shortly, after the like transition period expires, it is again invisible like in the mathematical case simulation. This is illustrated by Figure 11, which is an enlarged segment of Figure 10 for $t$ between 0.15 and 0.5 (sec). Evasion of the occurrence is achieved by appropriate choice of the sliding subspace and $\sigma$-neighborhood parameters through vector $c$ and parameter $\sigma$, as well as the state space area, where the linear model is very adequate.

It has already been pointed out that exponential stability is a higher quality stability property compared to "common" stability in Lyapunov's sense (in this case it refers to global asymptotic stability of a set-sliding subspace $S$ ). For that, it is 
natural that the sufficient conditions of Theorem 15 are more rigorous compared to the conditions of "common" stability. The conditions of Theorem 15 are indirectly applicable in the control of real-world processes through control law of Theorems 17 and 18, for these control laws were derived from the conditions of Theorem 15. Real experiment with control of DC servo motor is control of real-world process. In the way it is confirmed that the stability conditions of Theorem 15 are applicable in the control processes in practice.

To resume, in both ideal simulation case and in experimental application, the proposed new developed algorithm, in the paper, guarantees exponential stability of $S$, and no chattering in sliding mode regime. Despite the existence of all upper stated nonidealities, in the case of real application, by the proper parameters choice, the system motion is such that distance of working point from $S$ is always under proposed exponential envelope, as it could be seen in provided experiment.

\section{Conclusion}

In the paper, discrete-time type of VSCS with sliding modes is considered, related to the linear time-invariant plant of second order. The fuzzy exponential stabilizing state feedback control algorithm, so with prescribed approaching to the sliding subspace velocity estimate, is developed strictly by Lyapunov's second method and with chattering free sliding mode. Also, application of the proposed ideal cases algorithm, in practice, is considered and discussed through the experimental example. It shows that experiment is quite consistent with mathematical ideal cases.

\section{Conflict of Interests}

The authors declare that there is no conflict of interests regarding the publication of this paper.

\section{References}

[1] Z. Bucevac, "Lyapunov's method approach in a stabilizing control algorithm design for digital discrete VS systems with sliding modes-linear plant case," in Proceedings of the 7th International Symposium Modelling, Identification and Control ( IASTED '88), Grindelwald, Switzerland, 1988.

[2] G. Bartolini, A. Ferrara, and V. I. Utkin, "Adaptive sliding mode control in discrete-time systems," Automatica, vol. 31, no. 5, pp. 769-773, 1995.

[3] S. Hui and S. H. Żak, "On discrete-time variable structure sliding mode control," Systems \& Control Letters, vol. 38, no. 4-5, pp. 283-288, 1999.

[4] A. Bartoszewicz, "Discrete-time quasi-sliding-mode control strategies," IEEE Transactions on Industrial Electronics, vol. 45, no. 4, pp. 633-637, 1998.

[5] W. Gao, Y. Wang, and A. Homaifa, "Discrete-time variable structure control systems," IEEE Transactions on Industrial Electronics, vol. 42, no. 2, pp. 117-122, 1995.

[6] X. Yu and O. Kaynak, "Sliding-mode control with soft computing: a survey," IEEE Transactions on Industrial Electronics, vol. 56, no. 9, pp. 3275-3285, 2009.
[7] Y. Zheng and Y. Jing, "Approximation law for discrete-time variable structure control systems," Journal of Control Theory and Applications, vol. 4, no. 3, pp. 291-296, 2006.

[8] G. Golo and C. Milosavljević, "Robust discrete-time chattering free sliding mode control," Systems \& Control Letters, vol. 41, no. 1, pp. 19-28, 2000.

[9] H. Ying, "Theory and application of a novel fuzzy PID controller using a simplified Takagi-Sugeno rule scheme," Information Sciences, vol. 123, no. 3, pp. 281-293, 2000.

[10] G. Feng, "A survey on analysis and design of model-based fuzzy control systems," IEEE Transactions on Fuzzy Systems, vol. 14, no. 5, pp. 676-697, 2006.

[11] H. O. Wang, K. Tanaka, and M. F. Griffin, "An approach to fuzzy control of nonlinear systems: Stability and design issues," IEEE Transactions on Fuzzy Systems, vol. 4, no. 1, pp. 14-23, 1996.

[12] H. K. Lam, F. H. F. Leung, and P. K. S. Tam, "Stable and robust fuzzy control for uncertain nonlinear systems," IEEE Transactions on Systems, Man, and Cybernetics Part A: Systems and Humans, vol. 30, no. 6, pp. 825-840, 2000.

[13] R.-E. Precup, M. L. Tomescu, M.-B. Rdac, E. M. Petriu, S. Preitl, and C.-A. Dragoş, "Iterative performance improvement of fuzzy control systems for three tank systems," Expert Systems with Applications, vol. 39, no. 9, pp. 8288-8299, 2012.

[14] D. H. Lee, Y. H. Joo, and M. H. Tak, "Local stability analysis of continuous-time Takagi-Sugeno fuzzy systems: a fuzzy Lyapunov function approach," Information Sciences, vol. 257, pp. 163-175, 2014.

[15] R. Palm, "Robust control by fuzzy sliding mode," Automatica, vol. 30, no. 9, pp. 1429-1437, 1994.

[16] C.-L. Chen and M.-H. Chang, "Optimal design of fuzzy slidingmode control: a comparative study," Fuzzy Sets and Systems, vol. 93, no. 1, pp. 37-48, 1998.

[17] J. C. Wu and T. S. Liu, "A sliding-mode approach to fuzzy control design," IEEE Transactions on Control Systems Technology, vol. 4, no. 2, pp. 141-151, 1996.

[18] S.-W. Kim and J.-J. Lee, "Design of a fuzzy controller with fuzzy sliding surface," Fuzzy Sets and Systems, vol. 71, no. 3, pp. 359367, 1995.

[19] G. Feng, S. G. Cao, N. W. Rees, and C. K. Chak, "Design of fuzzy control systems with guaranteed stability," Fuzzy Sets and Systems, vol. 85, no. 1, pp. 1-10, 1997.

[20] F. Qiao, Q. M. Zhu, A. Winfield, and C. Melhuish, "Fuzzy sliding mode control for discrete nonlinear systems," Transactions of China Automation Society, vol. 22, no. 2, pp. 311-315, 2003.

[21] S. H. Cho, "Robust discrete time tracking control using sliding surfaces," KSME Journal, vol. 9, no. 1, pp. 80-90, 1995.

[22] V. I. Utkin, Sliding Modes in Control and Optimization, Communications and Control Engineering Series, Springer, Berlin, Germany, 1992.

[23] H. Ying, W. Siler, and J. J. Buckley, "Fuzzy control theory: a nonlinear case," Automatica, vol. 26, no. 3, pp. 513-520, 1990. 

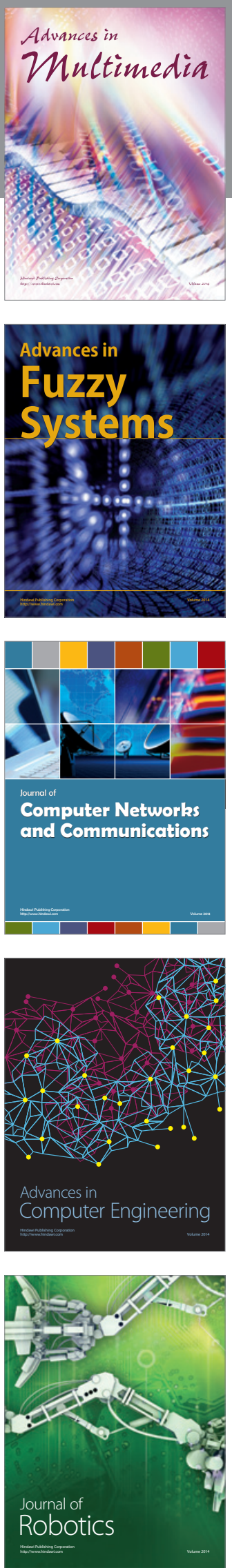

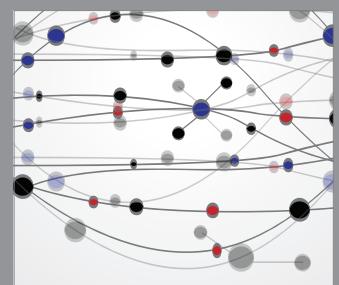

The Scientific World Journal
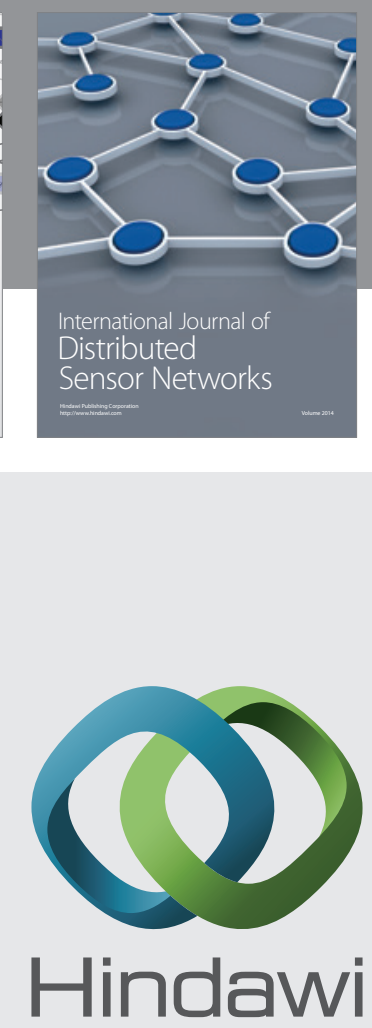

Submit your manuscripts at

http://www.hindawi.com
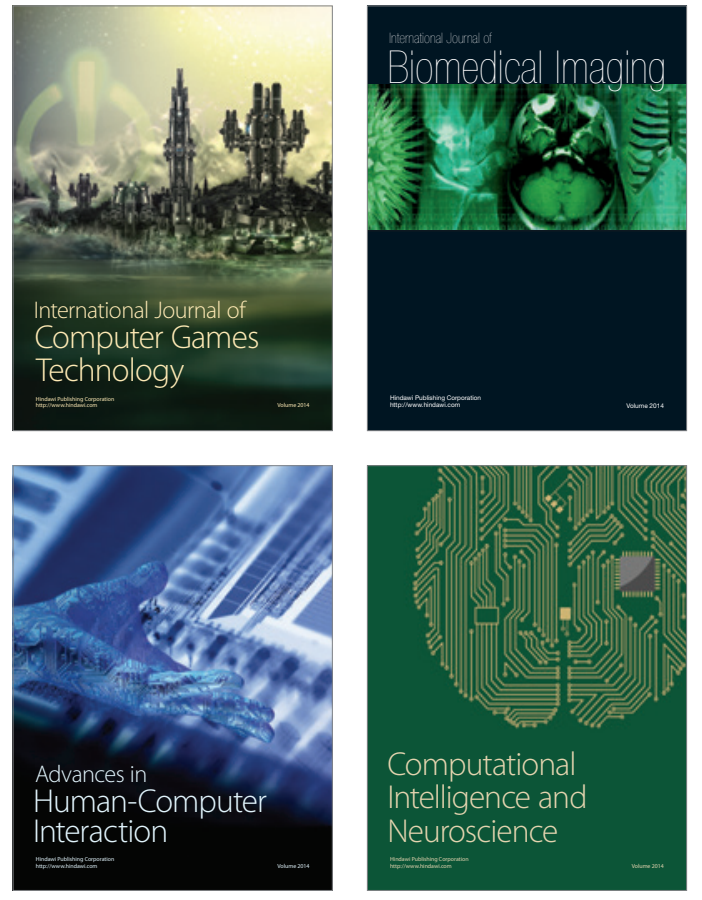
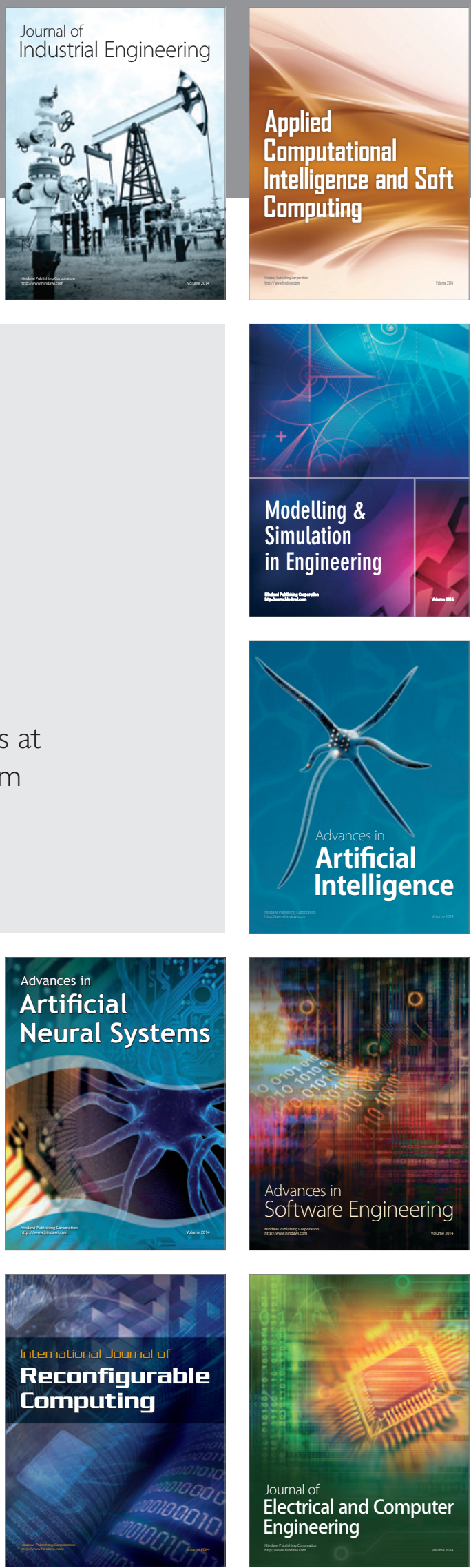